au niveau de l'amygdale, une structure déterminante dans l'expression de la peur. L'amygdale reçoit préférentiellement des projections en provenance de glomérules localisés dans la partie dorsale du bulbe. Ces glomérules dorsaux partagent la caractéristique de répondre à des odeurs qui suscitent des comportements d'évitement, comme l'odeur de prédateur ou de nourriture avariée [9]. Si la précision d'un tel câblage au niveau de l'amygdale contraste avec la dispersion et le caractère combinatoire des projections corticales, elle trouve un certain sens sur le plan adaptatif en raison de la forte valeur de survie des réponses comportementales aux odeurs signalant un danger. De manière intéressante, des schémas de connectivité semblables existent dans le système olfactif de la drosophile, où les réponses comportementales apprises et non apprises à des odeurs reposent sur des réseaux de projection respectivement aléatoires et ségrégés.

Encore une belle confirmation de l'unité du Vivant. $\diamond$

Olfaction: the cortex reshuffles the maps

\section{CONFLIT D'INTÉRÊTS}

Les auteurs déclarent n'avoir aucun conflit d'intérêts concernant les données publiées dans cet article.

\section{RéFÉRENCES}

1. Ghosh S, Larson SD, Hefzi H, et al. Sensory maps in the olfactory cortex defined by long-range viral tracing of single neurons. Nature $2011 ; 472$ : 217-20.

2. Miyamichi K, Amat F, Moussavi F, et al. Cortical representations of olfactory input by trans-synaptic tracing. Nature $2011 ; 472$ : 191-6.

3. Sosulski DL, Bloom ML, Cutforth T, et al. Distinct representations of olfactory information in different cortical centres. Nature $2011 ; 472$ : 213-6.

4. Mombaerts $P$, Wang F, Dulac $C$, et al. Visualizing an olfactory sensory map. Cell $1996 ; 87: 675-86$.

5. Sakano H. Neural map formation in the mouse olfactory system. Neuron $2010 ; 67: 530-42$

6. Rochefort N. Organisation dendritique et caractéristiques fonctionnelles des afférences visuelles sur les neurones corticaux. Med Sci (Paris) $2010 ; 26: 1009-12$.

7. Callaway EM. Transneural circuit tracing with neurotropic viruses. Curr Opin Neurobiol 2008 ; $18: 617-23$.

8. Poo C, Isaacson JS. Odor representations in olfactory cortex : 'sparse' coding, global inhibition and oscillations. Neuron $2009 ; 62: 850-61$

9. Kobayakawa K, Kobayakawa R, Matsumoto H, et al. Innate versus learned odour processing in the mouse olfactory bulb. Nature $2007 ; 450: 503-8$.

\title{
NOUVELle
}

\section{Le cannibalisme cellulaire par entose}

\section{Un nouveau mécanisme} d'aneuploïdie tumorale

Arnaud A. Mailleux, Matej Krajcovic, Michael Overholtzer

\section{Cannibalisme cellulaire et entose}

Le cannibalisme cellulaire dans les tissus cancéreux fut découvert à la fin du $\mathrm{XIX}$ siècle [1]. Contrairement au processus d'internalisation et d'élimination rapide de cellules mortes par les cellules phagocytaires dites «professionnelles », le cannibalisme cellulaire se caractérise par la présence d'une cellule vivante au sein d'une cellule hôte qui n'est pas une cellule phagocytaire professionnelle. De plus, la cellule internalisée n'est pas dégradée immédiatement par la cellule hôte. Le cannibalisme cellulaire peut être hétérotypique comme dans le cas d'un lymphocyte internalisé dans des cellules de mélanome ou dans des cellules épithéliales d'origine intestinale [1]. En revanche, l'entose, du grec entos (dedans), est une forme de cannibalisme cellulaire homotypique entre cellules épithéliales (Figures 1 et 2) au cours duquel la cellule internalisée va envahir activement la cellule hôte $[2,3]$.

Initialement, l'entose a été mise en évidence lorsque des cellules de lignées épithéliales d'origine mammaire étaient cultivées en suspension (Figure IA). Dans ces conditions, il y a perte d'engagement des récepteurs des protéines de la matrice extracellulaire de type intégrine, et les cellules forment des agrégats au sein desquels des cellules vivantes se trouvent encapsulées dans une vacuole à l'intérieur d'autres cellules (Figure I). Ce processus, déclenché par la perte de contact avec la matrice extracellulaire, met en jeu des forces contractiles générées
A.A. Mailleux : Université Paris Diderot, Sorbonne Paris Cité, Inserm U700, 75018, Paris, France.

M. Krajcovic M. Overholtzer :

Cell Biology Program, Memorial SloanKettering Cancer Center, New York 10065 , États-Unis; BCMB Allied Program, Weill Cornell Medical College,

1300 York Avenue, New York 10065, États-Unis.

par les jonctions adhérentes de type cadhérine connectées au réseau du cytosquelette d'actine intracellulaire (Figure 2A). Il est important de noter que la cellule internalisée est vivante au moment de l'entose et que la cellule hôte joue un rôle passif au cours de ce processus. Ce mécanisme d'internalisation homotypique contrôlé par les cadhérines permet de distinguer l'entose des autres processus de cannibalisme cellulaire [1-3]. Par la suite, la cellule internalisée est digérée lentement par les lysosomes de la cellule hôte dans la plupart des cas (Figure 2A). L'entose aboutit ainsi à l'élimination de la cellule envahisseuse. Cependant, l'étude minutieuse du destin de la cellule internalisée en vidéomicroscopie montre que celle-ci peut aussi se divi- 

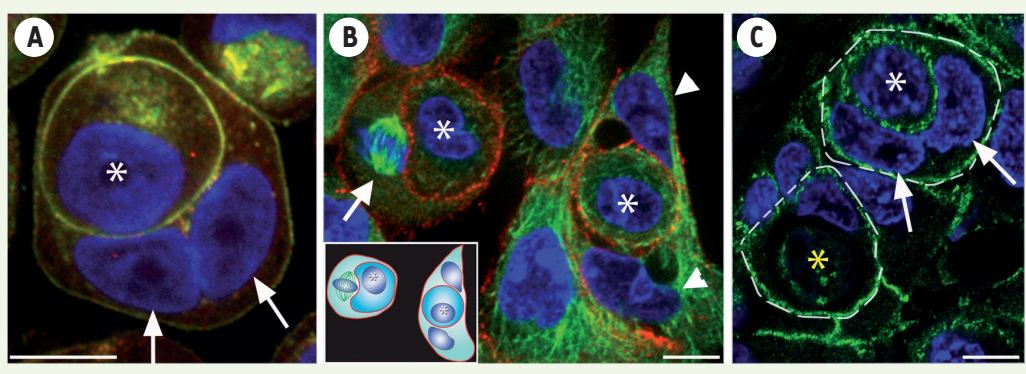

Figure 1. Images d'entose en microscopie confocale. A. Images d'entose dans des agrégats de cellules mammaires MCF-7 cultivées en suspension in vitro. Le marquage de la $\varepsilon$-cadhérine (vert) et de la $\beta$-caténine (rouge) marquent les contours cellulaires. Le DAPI (bleu) marque l'ADN des noyaux. La cellule hôte qui contient la cellule internalisée (astérisque) est binucléée (flèches blanches). B. Images d'entose de cellules mammaires MCF-10A cultivées sur lamelle (vert: $\alpha$-tubuline; rouge : $\beta$-caténine; bleu: DAPI). La cellule hôte à gauche est en cours de mitose (flèche blanche) alors que la cellule hôte à droite est binucléée (têtes de flèche blanches). Les cellules internalisées sont marquées d'un astérisque. C. Images d'entose au sein de tumeurs solides d'origine mammaire. Les figures d'entose sont marquées en pointillé (vert : $\beta$-caténine; bleu: DAPI). Une des cellules hôte est binucléée (flèches blanches). Les cellules internalisées sont marquées d'un astérisque. Noter que la cellule internalisée marquée d'un astérisque jaune est dégradée par la cellule hôte. Barre d'échelle : $10 \mu \mathrm{m}$.

ser au sein de l'hôte, voire même s'en échapper (Figure 2A). Ces observations soulignent bien que la cellule internalisée est vivante.

Ce processus de cannibalisme cellulaire par entose est aussi observé dans de nombreuses lignées cellulaires d'origine épithéliale in vitro ainsi que dans de nombreux tissus cancéreux chez l'homme [1, 3, 4]. Par exemple, des figures de cannibalisme cellulaire ayant les caractéristiques d'entose sont observées dans de nombreuses tumeurs d'origine épithéliale (Figure IC) ou dans des effusions pleurales d'origine métastatique. Ces figures sont caractérisées par la présence de jonctions adhérentes entre la cellule hôte et la cellule internalisée en l'absence d'attachement avec la matrice extracellulaire, et ce même dans les tumeurs solides.

\section{L'entose perturbe la division cellulaire} Récemment, il a été montré que l'entose pouvait aussi avoir des conséquences importantes sur la cellule hôte lorsque celle-ci se divisait [4]. Sur la base d'observations faites dans des cultures de cellules in vitro, sur des coupes de tumeurs solides et dans des cellules tumorales métastatiques isolées à partir d'effusion pleurale, il est apparu qu'une proportion importante des cellules hôtes étaient binucléées voir multinucléées (Figure 1). En effet, la présence d'une cellule internalisée par entose pouvait perturber la séparation du cytoplasme de la cellule hôte qui intervient en fin de division cellulaire pour former les deux cellules filles. En conséquence, la cellule hôte devenait binucléée. Ce processus de séparation appelé cytocinèse commence par la formation d'un sillon de division dans un plan perpendiculaire à l'axe du fuseau mitotique. Ce sillon va ensuite se resserrer pour séparer les deux cellules filles (Figure 2B). La constriction du sillon est contrôlée par un anneau contractile composé d'actine et de myosine. Dans un nombre élevé de cas, la cellule internalisée perturbait physiquement la formation et la contraction du sillon de la cellule hôte en cours de division cellulaire. Dans ce cas, la cellule internalisée doit se trouver exactement dans l'axe du plan de division (Figure 2B). En revanche, si la cellule internalisée se trouve dans un autre plan, la cytocinèse de la cellule hôte ne sera pas perturbée et les deux cellules filles pourront se séparer normalement (Figure 2B). D'un point de vue mécanistique, la présence d'une cellule internalisée dans le plan de clivage inhibait l'activation de l'anneau contractile du côté de la cellule internalisée. La perturbation physique et non génétique de la cytocinèse peut ainsi générer des cellules multinucléées [4]. Un processus comparable a été observé dans des cellules épithéliales ayant ingéré de longues fibres d'amiante. L'amiante a été impliqué dans le développement de cancers agressifs tels que le mésothéliome et certains cancers bronchiques. Dans le cas de l'amiante, les fibres pouvaient aussi bloquer physiquement la progression du sillon et la contraction de l'anneau au cours de la cytocinèse et faire échouer la séparation des deux cellules filles en fin de mitose [5].

\section{Entose, un facteur favorisant} I'aneuploïdie tumorale

Ainsi, le cannibalisme cellulaire par entose peut générer une cellule hôte multinucléée ayant un nombre anormal de chromosomes. Par la suite, les cellules hôtes binucléées (avec donc deux copies du génome) peuvent de nouveau se diviser et donner naissance à des cellules filles qui seront polyploïdes et porteuses de plus de deux copies de chaque chromosome [4]. Ces cellules polyploïdes à leur tour donneront naissance très probablement à de nouvelles cellules filles présentant des aberrations chromosomiques causées par la ségrégation anormale des chromosomes surnuméraires. Ce type d'aberration chromosomique appelée aneuploïdie est observé dans de nombreux cancers, en particulier aux stades avancés de la maladie. Selon l'hypothèse actuelle, l'aneuploïdie favoriserait la croissance tumorale et l'acquisition de 
A

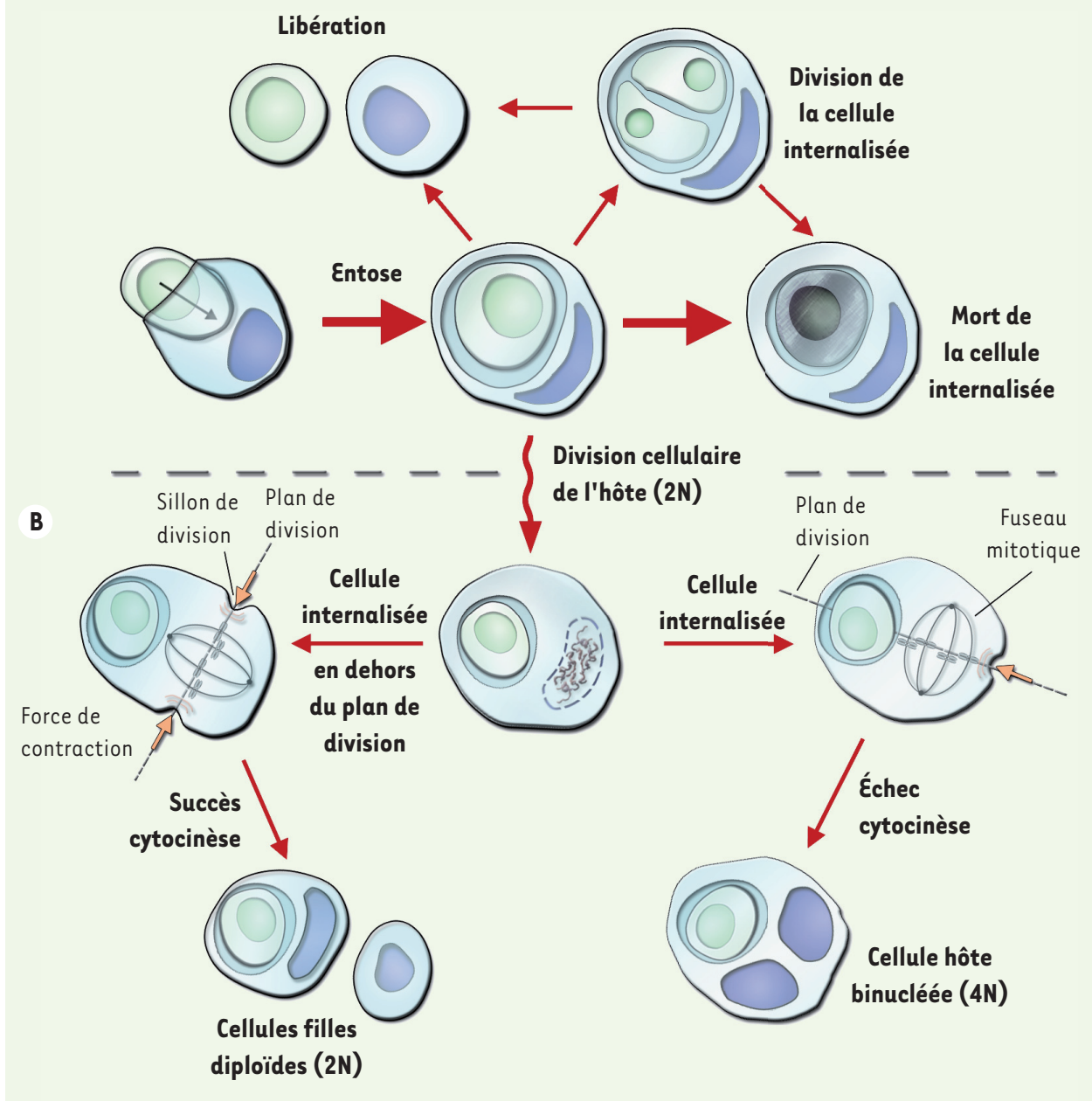

Figure 2. Représentation schématique de l'entose. A. une cellule (verte) va envahir activement (flèche) une autre cellule (bleue). La cellule internalisée soit sera dégradée par la cellule hôte, soit se divisera, soit s'échappera. B. Effets de la cellule internalisée sur la cellule hôte en cas de division cellulaire. La cellule internalisée peut perturber la séparation des deux cellules filles en fin de division de la cellule hôte et générer une cellule hôte aneuploïde binucléée (voir texte pour les détails).

résistance aux chimiothérapies par la perte de fragments de chromosomes porteurs de gènes suppresseurs de tumeurs ou en augmentant le nombre de copies d'oncogènes par l'acquisition de fragments de chromosomes supplémentaires. L'aneuploïdie tumorale est associée à un mauvais pronostic pour la survie du patient. Dans les cancers du sein, le nombre de figures d'entose augmentait avec la sévérité des tumeurs [4]. Ces observations suggèrent que le cannibalisme cellulaire par entose dans les tumeurs pourrait générer des cellules aneuploïdes. À l'heure actuelle, l'étude du rôle de l'aneuploïdie dans la croissance tumorale se base essentiellement sur des modèles génétiques chez la souris [6]. Avec l'entose, les chercheurs ont la possibilité d'étudier un processus naturel pouvant mener à l'aneuploïdie au cours de la croissance tumorale chez l'homme.

Que penser de l'impact du cannibalisme cellulaire par entose sur la croissance tumorale? Son rôle semble ambivalent: si il peut limiter la croissance tumorale en éliminant la cellule internalisée, il introduit aussi un risque non négligeable de perturbation du bon déroulement de la division cellulaire de la cellule hôte. L'entose peut en conséquence induire l'apparition de cellules tumorales aneuploïdes qui augmenteront l'instabilité génomique de la tumeur et potentiellement en favoriseront l'agressivité. Ainsi les questions de la régulation de l'entose in vivo et son impact sur la croissance tumorale in vivo restent encore sans réponse. $\diamond$

Cell cannibalism by entosis: a new pathway leading to aneuploidy in cancer

\section{RÉFÉRENCES}

\begin{abstract}
1. Overholtzer M, Brugge JS. The cell biology of cell-in-cell structures. Nat Rev Mol Cell Biol 2008 ; 9 : 796-809.

2. Mailleux AA, Overholtzer M, Brugge JS. L'entose, mort cellulaire par cannibalisme entre cellules tumorales. Med Sci (Paris) 2008 ; 24 : 246-8.

3. Overholtzer M, Mailleux AA, Mouneimne $G$, et al. A nonapoptotic cell death process, entosis, that occurs by cell-in-cell invasion. Cell 2007 ; 131 : 966-79.

4. Krajcovic M, Johnson NB, Sun $Q$, et al. A non-genetic route to aneuploidy in human cancers. Nat Cell Biol 2011 ; 13 : 324-30. 5. Jensen CG, Jensen LC, Rieder CL, et al. Long crocidolite asbestos fibers cause polyploidy by sterically blocking cytokinesis. Carcinogenesis 1996; 17 : 2013-21.

6. Ricke RM, van Ree JH, van Deursen JM. Whole chromosome instability and cancer: a complex relationship. Trends Genet 2008 ; $24: 457-66$.
\end{abstract}

\section{CONFLIT D'INTÉRÊTS}

Les auteurs déclarent n'avoir aucun conflit d'intérêts concernant les données publiées dans cet article.

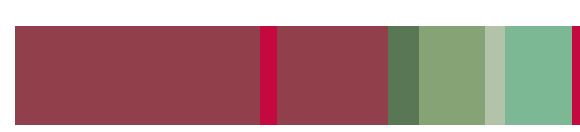

